\title{
Seismology for urban activity monitoring in Singapore under the impact of COVID-19
}

\author{
Yunyue Elita $\mathrm{Li}^{1 *}$, Enhedelihai Nilot ${ }^{1}$, Yumin Zhao $^{1}$, Gang Fang ${ }^{1}$ \\ ${ }^{1}$ Department of Civil and Environmental Engineering, National University of Singapore \\ ${ }^{1}$ All authors contributed equally \\ ${ }^{*}$ Corresponding E-mail: elita.li@ nus.edu.sg
}

Urban activities cause minute vibrations of the earth surface that can be detected by highly sensitive seismometers. In the island city-state Singapore, human activities have been dramatically altered since April 2020 by government measures aimed at suppressing the spread of COVID-19. By analyzing the high-frequency seismic signals, the impact of the pandemic and its corresponding mitigation measures were quantified as traffic flow at the exit of the National University of Singapore (NUS) and at the intersection between Pasir Panjang Port Terminal and West Coast Park, representing activities at nonessential workplaces, essential workplaces, and recreational areas. The anonymity of seismic data enabled an unprecedented spatial and temporal resolution that is pivotal to understand the heterogeneity and evolution of pandemic responses in different sectors of an urban society. The rich information extracted from seismic data provide a unique opportunity for realtime activity monitoring and dynamic policy making to ensure a successful pandemic mitigation while minimizing negative impacts on urban lifestyle. 


\section{Introduction}

The ongoing COVID-19 pandemic has changed human life profoundly since its initial outbreak in December 2019 (1). To mitigate the virus spread, most countries across the world have implemented lockdown measures of various degrees of stringency to reduce the reproduction rate of the virus (2). These measures have to strike a delicate balance between reduction of population mixing (e.g., strict work from home orders (3)), maintaining sufficient supply of essential goods and services (e.g., food (4) and health care (5)), and encouraging a healthy and productive lifestyle (e.g., allowing recreational activities (6)). Due to the high mobility and high population density, urban activities in megacities around the world are not only the most severely impacted by the pandemic, but also the most consequential for the effectiveness of the mitigation measures (7-9). Urban society in megacities is highly heterogeneous in its functions, hence demonstrating very large variability in their temporal activity patterns (10). Therefore, monitoring and understanding the realtime changes of different sectors of an urban society is vital for policy making and dynamic pandemic management (11). However, current urban monitoring solutions using surveillance cameras and cell phone signals have introduced more concerns than reassurance due to potential intrusion of individual privacy $(12,13)$. Google and Apple have published anonymous, averaged statistical data based on individual usages in cities $(14,15)$, but by averaging over locations and populations, the inherent heterogeneities of urban activities are not sufficiently preserved. As a result, there is yet no established analysis of the impact of mitigation measures on different sectors of urban society.

Seismometers are sensitive to the minute vibrations caused by a wide range of natural activities such as earthquakes, volcano eruptions and ocean waves $(16,17)$, and human activities such as nuclear explosions, fracking, and construction (18-20). Recent development in stand-alone high-frequency seismometers greatly reduces the cost of individual sensors and the challenges 
associated with their deployment. When installed in an urban environment, seismometer signals are dominated by the road traffic (21-23). It has been observed that global seismic noise was significantly reduced due to the lockdown measures (24), and that the ambient noise level correlates well with the isolation index of the city (25). These human-induced noise recordings at different locations provide a unique tool to assess the dynamic impact of lockdown measures on urban life in different sectors. In this study, we analyze the high-frequency anthropogenic seismic noise and quantify the intensity of human activity at specific locations where the seismometers are deployed. The sensor deployment has a minimal intrusion on the environment, and the signal analysis is completely anonymous. Hence, we achieve an unprecedented spatial and temporal resolution without incurring privacy concerns.

\section{Data and Methods}

Singapore COVID-19 lockdown, called Circuit Breaker (CB) started on 8th April, 2020 with a target of $70 \%$ reduction in urban mobility. A two-phase opening was implemented on 1st June and 19th June, with mobility reduction targets at $50 \%$ and $40 \%$, respectively. We evaluated the effects of these measures based on seismic recordings at the high frequency band $(30-150 \mathrm{~Hz})$ installed at two locations, one at the roadside at the exit of the campus of the National University of Singapore, and the other at the geographical boundary between West Coast Park and Pasir Panjang Port Terminal (Fig. 1). At both locations, road traffic flow (motor vehicles) was estimated from the vertical-component seismic waveform data. In addition, running activities were also extracted from the horizontal-component of the seismic waveform data in the park. Temporal patterns and their reactions to the Singapore lockdown measures showed significant differences in different urban scenarios.

We extracted activity information from the direct vibration measurements of the seismometers. While average seismic energy at lower frequency band $(<30 \mathrm{~Hz})$ reflected an overall 
(a)

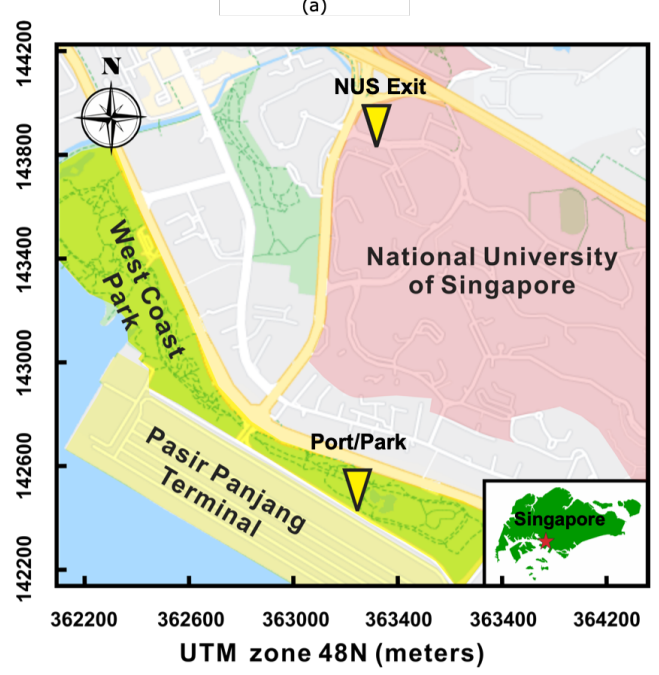

(b)
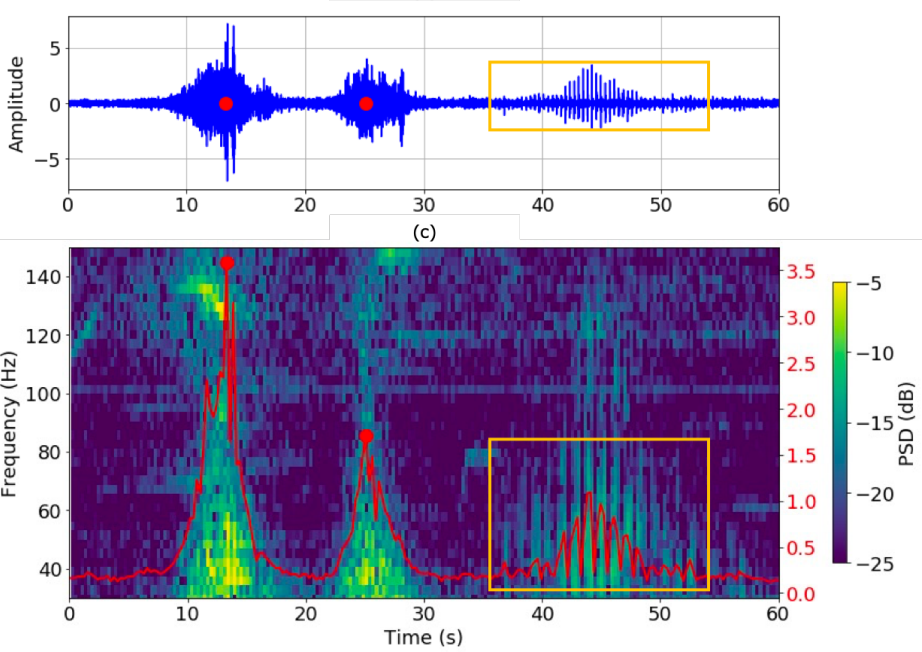

Figure 1: (a) Map of the study area. The two yellow triangles denote the locations of the two high-frequency SmartSolo seismometers, one at the exit of the campus of the National University of Singapore, the other at the intersection between Pasir Panjang Container Port Terminal and West Coast Park. The red star in the insert shows the location of the study area on the island of Singapore. (b) Example of 60 seconds of data recorded at the Port/Park location around 21:22:00 18th April 2020 (GMT+8). Data were filtered between 30 and $150 \mathrm{~Hz}$, and clearly showed two motor vehicles (red dots) and one runner (orange box) signals standing out from the background. (c) Spectrogram of the time domain signal in (b). The motor vehicles generated short-duration vibrations in the whole frequency band. The runner generated longduration periodic spiking signals. The red line denotes the summed PSD between 40 and 100 $\mathrm{Hz}$, from which the single large peaks were identified as motor vehicles and the periodic event was identified as a runner.

activity level within a few kilometers around the seismometer, the high frequency $(>30 \mathrm{~Hz})$ seismic data were primarily dominated by energy pulses generated within a few meters (Fig. S5 and S6). We identified events on the spectrograms of the time domain data records. Each motor vehicle generated a short burst of vibrations that appear across the whole frequency band for about two seconds. Each runner, in contrast, generated periodic spiking signals corresponding to the alternating footsteps. We designed specific algorithms to separate the runners from the motor vehicles at the Port/Park intersection (Supplementary Materials, Materials and Methods). 


\section{Results}

As a workplace primarily serving the urban educational activities within Singapore, the traffic pattern on NUS campus on weekdays closely reflected the working routine of urban residents and the mobility reduction targets enforced by the government mitigation measures (Fig 2). The trend of daily traffic count agreed very well with Google Mobility Report for workplaces (Fig 2b). Every day between 1:00 and 5:00, the average number of motor vehicles exiting NUS campus was below 10 per hour. During CB, the traffic exiting NUS campus on weekdays appeared uniform during the day hours (8:00 - 19:00). We observed that the decrease of traffic flow was immediate after $\mathrm{CB}$, while the increase was gradual since the strict restrictions were lifted. After Phase 2 opening, the weekday traffic started to show morning and evening peaks at 9:00 and 18:00, which was consistent with the traffic pattern before the pandemic. Although traffic flow over the weekend was much lighter compared to weekdays, it also increased gradually after the Phase 1 opening. Traffic flow exiting NUS campus increased around noon (11:00 to 13:00) due to the continuous service of on-campus dinning centers, University Health Center, and National University Hospital.

Traffic flow at the Port (Fig 3) showed a relatively constant flow during CB and different phases of opening. Most of the traffic identified at this location were heavy trucks that transported containers in and out of the port terminal. Truck drivers worked on 12-hour shifts and served the import and export business 24 hours a day, 7 days a week. Nonstop traffic flow was observed with an average of 50 motor vehicles per hour during the quietest time each day from $22: 00$ to $3: 00$. Significantly more drivers chose to work during day time shifts $(5: 00$ to $17: 00)$ than during night time shifts. Since the drivers are required to work at least one day during

the weekend, the peak volume hours were shorter (6:00 to 15:00) while the peak flow (around 300 vehicles per hour) was the same as the weekdays. The disparities between the government 

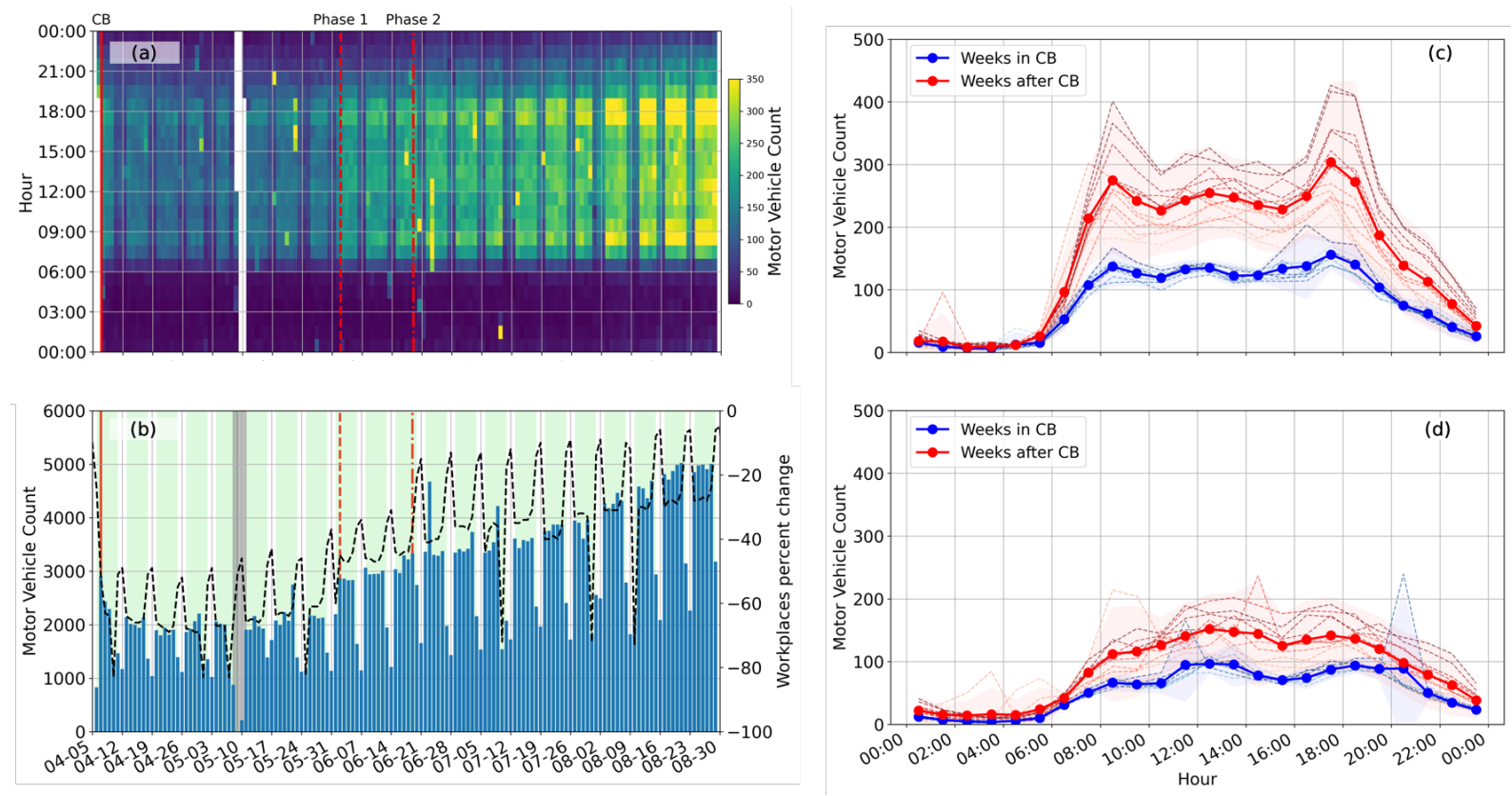

Figure 2: Traffic flow detected by the seismometer at NUS Campus. (a) Number of motor vehicles detected each hour from 8th April to 30 Aug, 2020. (b) Daily motor vehicle count. The black dash line denotes the percentage change at workplaces from the Google Mobility Report. In (a) and (b), the solid red line denotes the start of CB, while the dash and the dotteddash red lines denote the start of Phase 1 and 2 opening, respectively. (c) Hourly motor vehicle count averaged over weekdays. (d) Hourly motor vehicle count averaged over weekends. In (c) and (d), Data recorded during (Week 1 - 8) and after (week 9 - 21) CB are denoted respectively by blue and red dashed lines, with lighter shade denoting earlier time. The thick solid red and blue lines denote the mean vehicle count over the corresponding period and the shaded area covers the range of twice the standard deviations above and below the mean. 

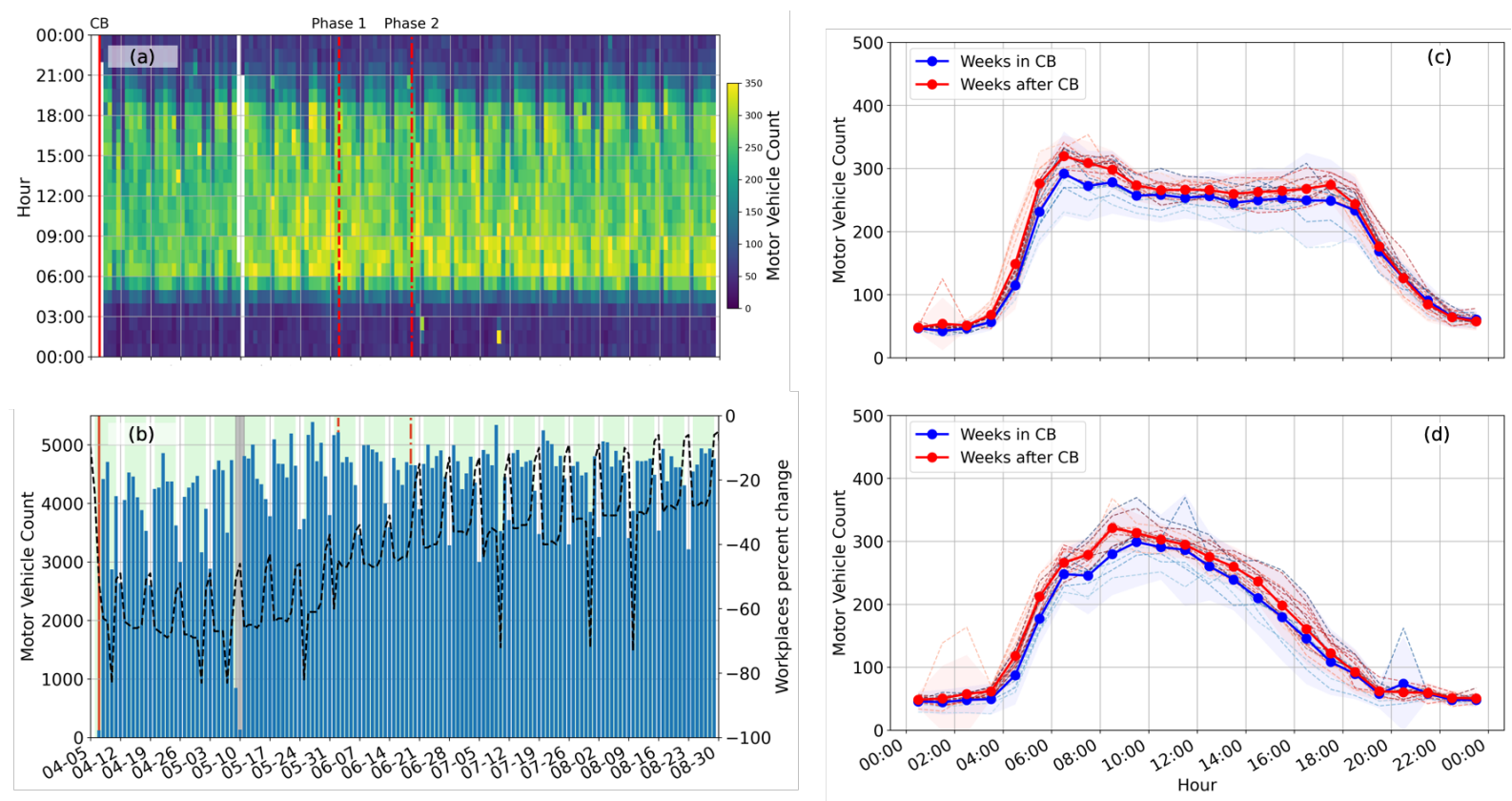

Figure 3: Traffic flow detected by the seismometer at Pasir Panjiang Port. Subplots and legends are the same as in Fig. 2.

mitigation measures and the traffic flow count reflected the function of the port, an interface between the island of Singapore and the rest of the world. Much of the port service is essential, since Singapore lacks land space and natural resources and relies heavily on international trade for its food, energy, and medical equipments. It is evident that the international port maintained its activities during $\mathrm{CB}$ and two phases of opening to provide essential services to Singapore.

The seismometer inside the West Coast Park allowed us to investigate runners' activities and to understand the workout preferences of urban residences (Fig 4). There were two consistent peaks in the running events, one in the morning (around 8:00) and the other in the evening (around 19:00). This could be a combined effect of working hours and the tropical climate. While the morning peak maintained a similar magnitude, the evening peak runner count decreased significantly as soon as the CB measures were lifted, especially on the weekends. Markedly different from the Google Mobility Report for Parks - which provided an over- 

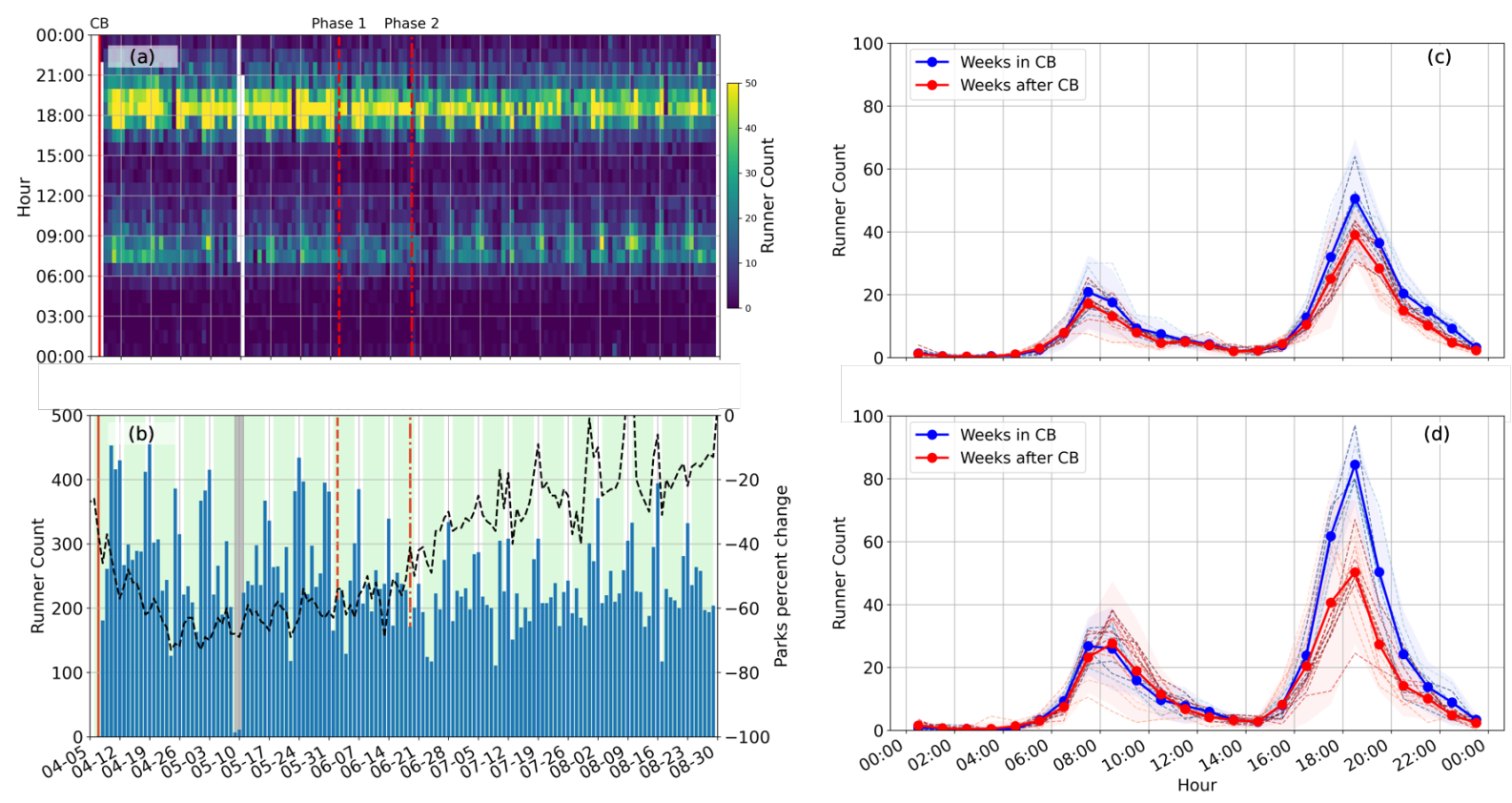

Figure 4: Runner count detected by the seismometer at West Coast Park. Subplots and legends are the same as in Figs 2 and 3.

all estimation for all park visitors - the runner count detected by the seismometer specifically accounts for the population who engaged in high-intensity physical activity. During $\mathrm{CB}$, an overwhelming portion of the urban residences were required to stay at home and/or work from home. These tight restrictions clearly promoted participation in high-intensity physical activity, particularly on weekend evenings. As soon as the majority of the workforce started to go back to the workplace, the peak number of weekend evening runners dropped by around $30 \%$, despite an increase in the number of overall park visitors. The relatively stable morning peak suggests that the morning runners are more disciplined and less influenced by the pandemic and corresponding measures. 


\section{Discussion}

Strict lockdown measures have been, and continue to be implemented in many countries around the world to reduce the human contact and suppress the spread of COVID-19 within local communities (2). Enforcing such measures universally requires a large amount of resources when most of the social attention is directed to directly combating COVID-19. If the measures are not fully respected, however, their effectiveness can be rather limited, which may lead to more infections and deaths that are avoidable, as well as prolonged lockdowns that are not practical both economically and psychologically (26). Compared to conventional solutions using surveillance cameras, cell phones, or other wearable GPS-enabled devices, our study achieved an unprecedented monitoring resolution for urban activities due to the anonymity of seismic recordings. When the public is less concerned about privacy intrusion, it will be more willing to participate in similar real-time statistical social studies $(12,27)$, which are crucial for quantitative evaluation of public compliance with the restrictions, optimizing law and regulation enforcements, and dynamic policy making (28).

Traffic activity extracted at the exit of NUS clearly demonstrated that the CB measures were dutifully observed and that the targets of each stage were successfully met. We estimated a different temporal evolution in work activity during working hours compared to the Google Mobility Report for Workplaces, whose measurements could have resulted from mixing the categories of essential and non-essential workplaces, an average between day and night, and an average across many geospatial locations (14). Our results agree well with the mitigation result that the number of community transmitted cases of COVID-19 in Singapore was reduced to single digits before Phase 2 opening (29). Strict compliance of urban residents with regulations enabled safer re-opening of local economic activities and better urban experiences during and after the island-wide lockdown. 
Data collected at the port provided a reference for the activities in the essential service sectors, in particular the construction sector which involves more than $95 \%$ of the confirmed infection cases in Singapore (30). The traffic activities at the port showed that essential service sector experienced an increase in workload during the second half of CB. To prevent high infection rates and fast spread of the virus, we suggest that better personal protection equipments and shift arrangements should be put in place while the essential workers maintain the lifeline of the Singapore island (31).

Due to the special geographical location and the severe demand of the highly urbanized society of Singapore, the traffic flow estimated at the Pasir Panjang Container Terminal Port also provided a glimpse of the international supply chain during the global pandemic of COVID19. Despite the global impact of the pandemic and many concerns about the disruption to the global food security (4), the international trade and supply were not immediately broken for the essentials such as food, energy, and medical supplies. This demonstrated continuous global connectivity and support under such an intense stress test, which made a land-scarce country like Singapore functional during the pandemic (32).

Although CB disrupted the work habits of most urban residents, it did not drastically change the cycle of their recreational habits. CB in Singapore promoted evening workouts, especially during weekends. The number of evening runners slightly increased towards the later period of $\mathrm{CB}$, which suggests a gradual increase of public concern towards maintaining active and healthy habits. This concern could have resulted from a gradual development of psychological fatigue from lockdown measures $(6,33)$. The increased running activity stressed the importance of accessibility to open green areas in an urban environment during a time of crisis (34). As soon as Phase 1 opening started on 1st June, the peak number of runners decreased and the peak time of running activities shifted to a later time. In particular, a large decrease in the Sunday evening runners was observed after Phase 2 opening, which suggests that a significant 
psychological strain was placed when people expect the start of a new working week. These data are paramount to sociological studies of human behaviour in order to support government mitigation policy and individual response to the pandemic (35-37).

\section{References}

1. C. Sohrabi, et al., International Journal of Surgery 76, 71 (2020).

2. N. Islam, et al., BMJ 370 (2020).

3. R. M. Anderson, H. Heesterbeek, D. Klinkenberg, T. D. Hollingsworth, The Lancet 395, 931 (2020).

4. J. E. Hobbs, Canadian Journal of Agricultural Economics 68, 171 (2020).

5. P. Dashraath, et al., American Journal of Obstetrics and Gynecology 222, 521 (2020).

6. G. Hall, D. R. Laddu, S. A. Phillips, C. J. Lavie, R. Arena, Progress in Cardiovascular Diseases PMC7194897 (2020).

7. Q. Lin, et al., International Journal of Infectious Diseases 93, P211 (2020).

8. C. T. CDC, et al., MMWR Morbidity and Mortality Weekly Report 69 (2020).

9. S. Mahato, S. Pal, K. G. Ghosh, Science of the Total Environment p. 139086 (2020).

10. N. J. Yuan, et al., IEEE Transactions on Knowledge and Data Engineering 27, 712 (2014).

11. T. K. Das, A. A. Savachkin, Y. Zhu, IIE Transactions 40, 893 (2008).

12. S. Gerke, C. Shachar, P. R. Chai, I. G. Cohen, Nature Medicine pp. 1-7 (2020).

13. N. Oliver, et al., Science Advances 6 (2020). 
14. A. Aktay, et al., Google COVID-19 Community Mobility Reports: Anonymization Process Description (version 1.0) (2020).

15. Apple, Apple Mobility Trends Reports (2020). Accessed: 2020-07-30.

16. J. N. Brune, J. Oliver, Bulletin of the Seismological Society of America 49, 349 (1959).

17. R. K. Cessaro, Bulletin of the Seismological Society of America 84, 142 (1994).

18. R. P. Massé, Bulletin of the Seismological Society of America 71, 1249 (1981).

19. L. House, Geophysical Research Letters 14, 919 (1987).

20. G. Fang, Y. E. Li, Y. Zhao, E. R. Martin, Geophysical Research Letters 47, e2019GL086115 (2020).

21. N. Riahi, P. Gerstoft, Geophysical Research Letters 42, 2674 (2015).

22. J. Díaz, M. Ruiz, P. S. Sánchez-Pastor, P. Romero, Scientific Reports 7, 1 (2017).

23. Y. Zhang, Y. E. Li, H. Zhang, T. Ku, Geophysics 84, B169 (2019).

24. T. Lecocq, et al., Science 369 (2020).

25. F. L. Dias, et al., Geophysical Research Letters p. e2020GL088748 (2020).

26. T. May, BMJ 370 (2020).

27. L. Barkhuus, A. K. Dey, Interact (Citeseer, 2003), vol. 3, pp. 702-712.

28. M. Gilbert, M. Dewatripont, E. Muraille, J.-P. Platteau, M. Goldman, Nature Medicine 26, $643(2020)$

29. W. C. Lee, C. Y. Ong, Public Health 185, 15 (2020). 
30. N. Bagdasarian, D. Fisher, BMC Medicine 18, 1 (2020).

31. J. Woo, Policy and Society 39, 345 (2020).

32. G. B. Gregorioa, R. C. Ancog, Asian Journal of Agriculture and Development 17, 1 (2020).

33. R. P. Rajkumar, Asian Journal of Psychiatry p. 102066 (2020).

34. Z. Venter, D. Barton, V. Gundersen, H. Figari, M. Nowell, Environmental Research Letters (2020).

35. J. J. Van Bavel, et al., Nature Human Behaviour pp. 1-12 (2020).

36. C. Ho, C. Chee, R. Ho, Ann Acad Med Singapore 49, 1 (2020).

37. S. X. Zhang, Y. Wang, A. Rauch, F. Wei, Psychiatry Research p. 112958 (2020).

\section{Acknowledgments}

The authors acknowledge Cambridge Sensing Pte Ltd. for its financial support of this research. The authors acknowledge Huaigu Tang and Bei Li for their assistance in deploying the seismometers. The authors acknowledge Professor Arthur Cheng at NUS and Professor Ruben Juanes at MIT for their suggestions to improve the manuscript. YEL acknowledges Mr. Jin Hou for valuable discussions about the port functions and their relations to the economic activities within the island of Singapore.

\section{Supplementary materials}

Materials and Methods

Supplementary Text

Figs. S1 to S7 


\section{Tables S1}

References (1) 